\title{
Continuous Antibiotic Fermentation-Design of a 20 litre, Single-stage Pilot Plant and Trials with Two Contrasting Processes*
}

\author{
M. C. BARTLETT $†$ and PHILIPP GERHARDT \\ Department of Bacteriology, The University of Michigan, Ann Arbor, \\ Michigan.
}

Summary. The feasibility of producing antibiotics by single-stage continuous fermentation was explored by means of a specially designed pilot plant. Both the chloramphenicol and penicillin processes appeared adaptable to such an operation. At dilution rates of $\mathbf{1} \cdot \mathbf{0}$ and 0.5 volume changes per day respectively, yields of from $\frac{1}{4}$ to $\frac{1}{2}$ of the maxima obtained in batch operation were maintained in the steady-state for more than 2 weeks.

\section{Introduction}

Although the potential advantages of continuous fermentation are widely recognized and the theoretical basis is reasonably established, industrial use of the operation has been restricted almost entirely to yeast and yeast products, vinegar, and sewage treatment processes, all of which yield bulk products and depend for their advantageous operation on a highly restrictive growth situation.12 Future applications of continuous culture, as judged by current trends in research and development, ${ }^{3}, 4$ will probably extend into the production of fine chemicals and into fermentations where culture purity is much more precarious.

One such application of considerable potential importance is in antibiotic production. Since the open literature carried many but only brief notes of progress in this area (see later), we undertook a study to determine the feasibility of continuous antibiotic fermentation. This report describes the design of a $20 \mathrm{l}$,

* Preliminary reports of this work have been presented orally at the VIIth International Congress for Microbiology in August, 1958, and at the 134th American Chemical Society Meeting in September, 1958.

$\dagger$ Present address: Abbott Laboratories, North Chicago, Illinois. 
single-stage pilot plant and the outcome of fermentation trials of two contrasting antibiotic processes, chloramphenicol and penicillin.

\section{Pilot Plant Design and Operation}

We sought to design a fermentation unit which would be as simple as possible and potentially capable of scale-up to existing production equipment. The size, $20 \mathrm{l}$. working capacity, was consistent with usual practice and with the requirements of sampling and adequate feed pumping. The materials of construction were chosen with a view not only to economy but to their specific suitability. For instance, black iron pipe was used for air and steam lines, but the fermentor vent line was constructed of stainless steel because the surging of liquid within this line might otherwise build up high concentrations of iron in the culture. The fermentor and sterilizer vessels were made of Inconel-clad stainless steel. Feed lines were gum-rubber tubing.

A wide-angle view of the installation at one stage of its design is shown in Fig. 1, and a schematic diagram in Fig. 2. 'The feed

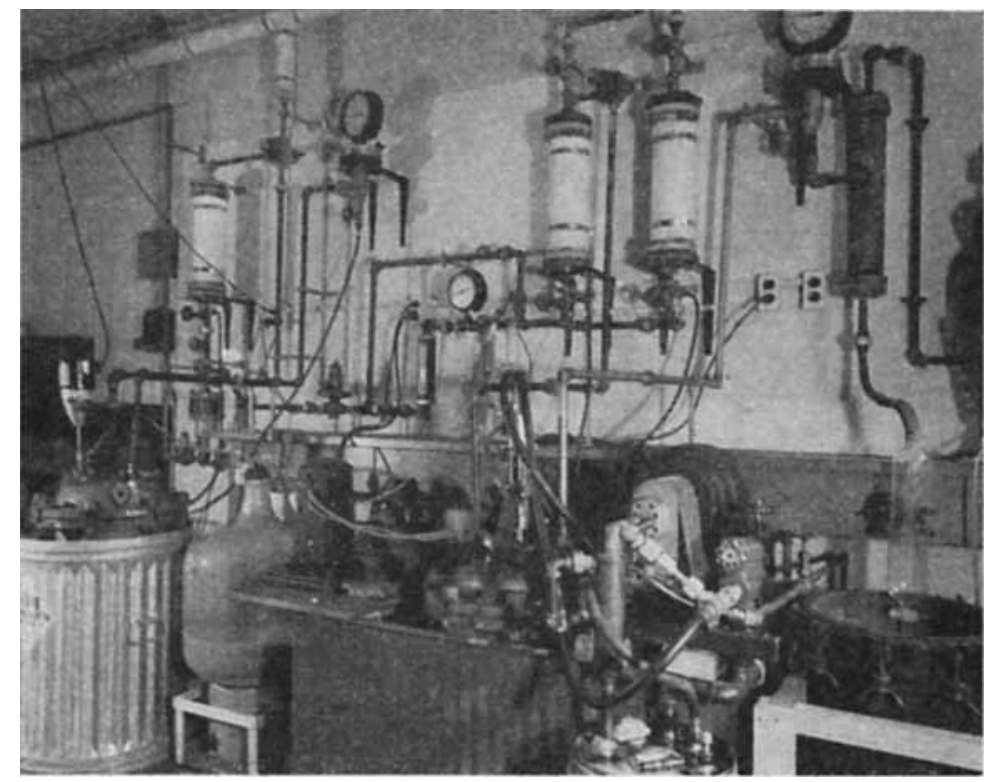

Fig. 1. Wide-angle view of pilot plant installation 


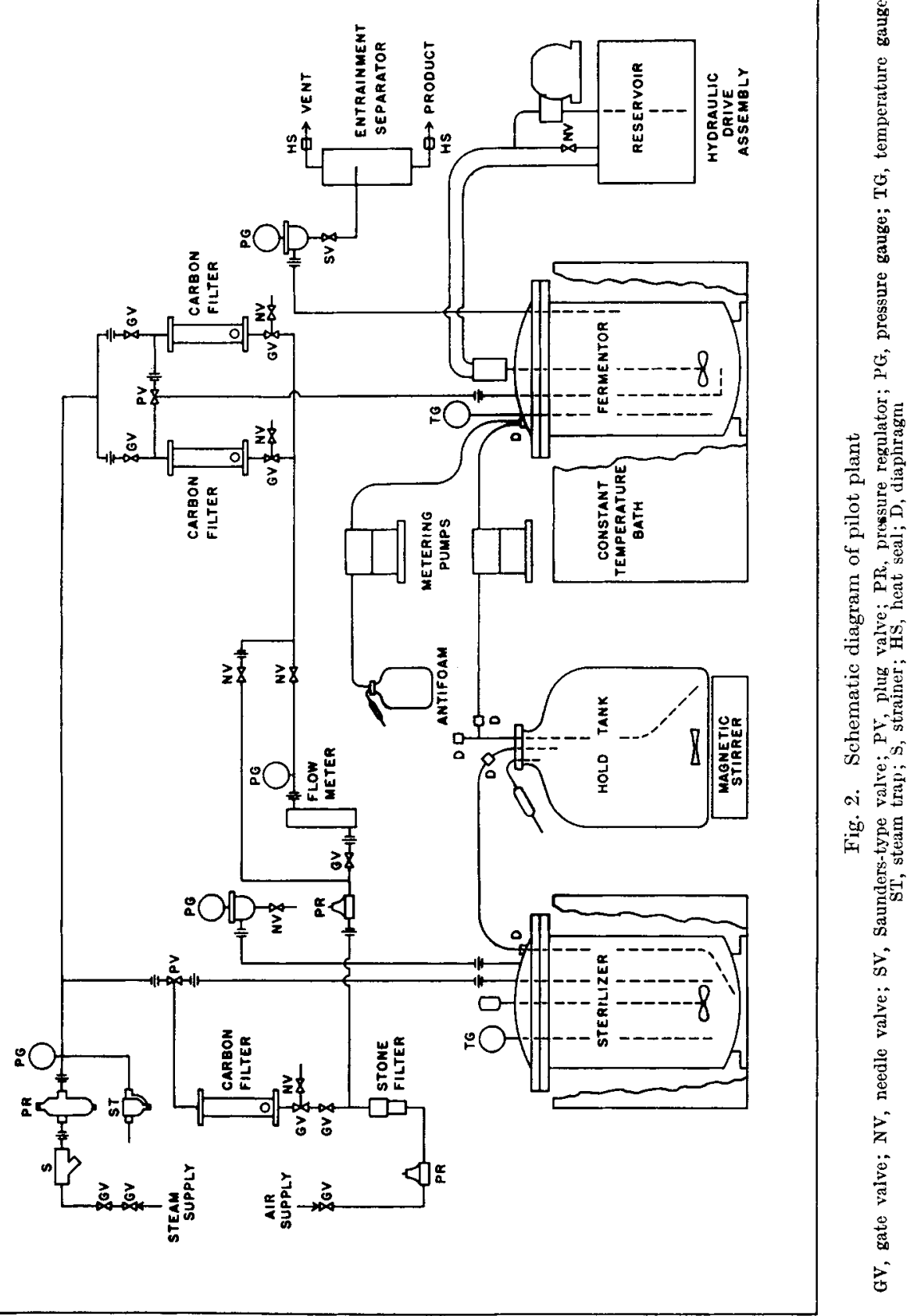


system was operated by first preparing a batch of medium and cooking it under pressure in the sterilizer vessel. The medium then was transferred by means of differential air pressure to the separately sterilized hold tank, a magnetically stirred, 13 gal Pyrex carboy. From this reservoir, the sterile medium was continuously metered into the fermentor by a peristaltic-action Sigma pump. Large hypodermic needles in the connecting feed lines and self-sealing rubber diaphragms fitted to the vessels permitted isolating the hold tank and fermentor from the sterilizer and also replacing the feed lines if necessary during operation.

The air supply to the fermentor was successively stone-filtered, metered, and sterilized by passage through an activated-carbon filter. Duplicate insulated filters (Fig. 3) were piped so as to

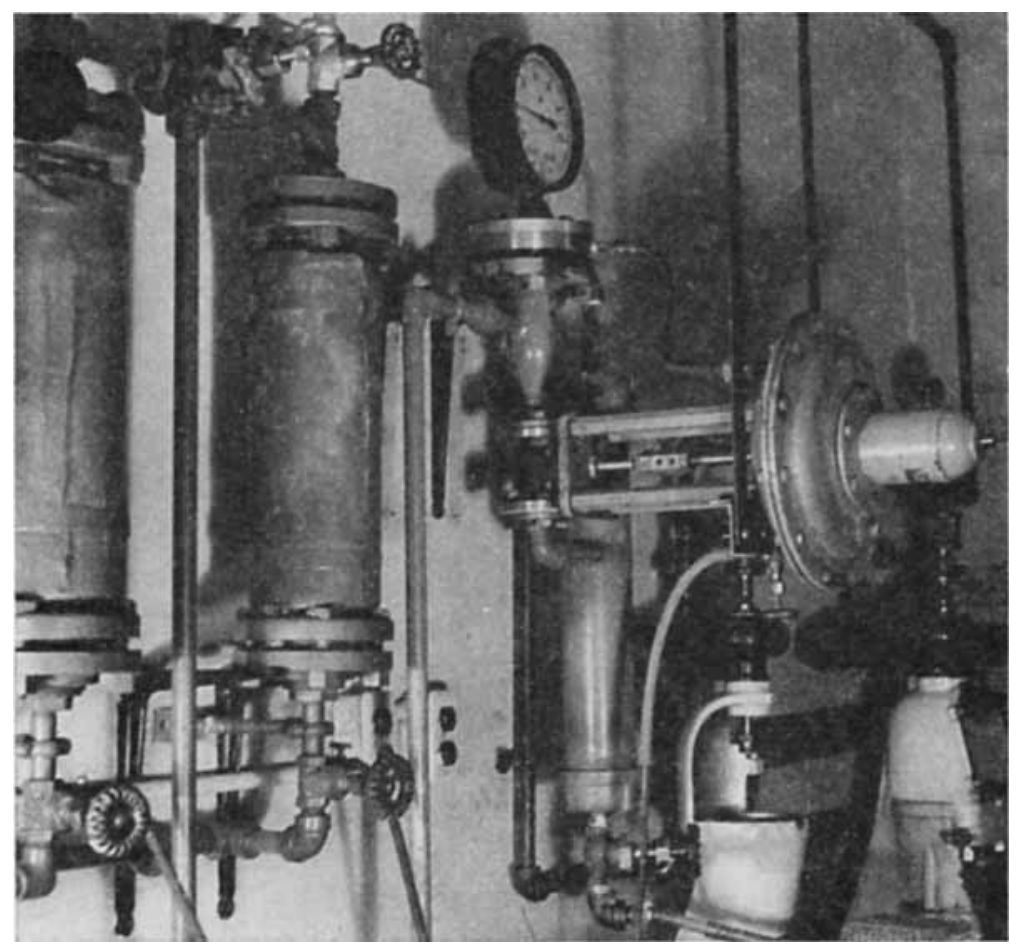

Fig. 3. Close-up view of air filters (left), back-pressure regulating unit (centre), and entrainment separator (behind and below regulator) 
allow alternate operation and steam sterilization on a 3-day cycle. The problem of occasional combustion of the carbon packing was solved by adding stainless steel wool to dissipate localized heat and by inspecting the packing through plugs in the bottom of the filters. The sterile air was passed into the contents of the fermentor through a $\frac{3}{8}$ in. stainless steel pipe opening $\frac{1}{2}$ in. below the centre of the impeller.

The fermentor vessel was fitted with a diaphragm adapter at the top for inserting the feed lines, and also near the bottom for sampling the contents by means of a sterile hypodermic syringe and needle. The contents of the fermentor were kept at constant temperature by means of an electrical thermoregulator and saber-heating unit acting against a constant flow of cold water in the water bath. A hydraulically driven turbine positioned on top of the fermentor drove the agitator shaft and conventional four-bladed impeller. The agitator shaft entered the vessel through a stuffing box packed alternately with $\frac{1}{4}$ in. teflonimpregnated asbestos rings and $\frac{1}{2}$ in. teflon spacers; mechanical seals were also attempted but proved less satisfactory. With the possibility of agitator speeds up to $700 \mathrm{rev} / \mathrm{min}$ and with the fermentor equipped with four vertical baffles, fully adequate agitation could be attained.

Both air and product were withdrawn through a pipe that extended into the fermentor to the liquid level. Maintenance of a constant liquid level in the fermentor with such an air-lift system required close control of foaming and this was accomplished with the continuous addition of antifoam agent. A separate sterile reservoir and continuous feed line for antifoam agent were necessary in the penicillin process, but a stable silicone emulsion could be incorporated into the medium in the chloramphenicol process. A spring-loaded, back-pressure regulating valve and a product-air separator were located in the effluent system and are shown in Fig. 3. The pressure regulator maintained a positive pressure of about $\tilde{5} \mathrm{lb} / \mathrm{in}^{2}$ gauge in the fermentor independent of the rate of flow of medium or air. Electric heating tapes used as heat seals were located on the effluent air and product pipes to prevent possible backgrowth of contaminating micro-organisms along the pipe walls. 


\section{Chloramphenicol Fermentation}

Chloramphenicol appears to be ideally adapted to continuous fermentation in a single-stage unit, because the batch fermentation exhibits parallel growth and antibiotic formation and, in addition, is favourably characterized by only moderate $\mathrm{pH}$ changes, a fluid cell suspension, wide-spectrum effectiveness against contamination, and stable product. As potential disadvantages, the hyphae of the chloramphenicol-producing Streptomyces venezuelae tend to fragment and lyse during the latter stages of the batch growth cycle, and the organism is understood to have a moderately high frequency of reversion to mutants with low synthetic ability. Details of the batch chloramphenicol process have been published.23, 24 To our knowledge, continuous fermentation of this antibiotic has not been attempted, although there have been brief reports or patents on continuous fermentation of streptomycin, ${ }^{4-6}$ subtilin, ${ }^{11}$ and penicillin (see below); these are discussed more fully elsewhere. ${ }^{12}$

Assay procedures. Product samples were withdrawn directly from the fermentor and frozen until a number of assays for antibiotic content could be made together. Chloramphenicol was determined by the method of Glazko et al.13 The aromatic nitro group of chloramphenicol was quantitatively reduced to a primary amine, which was then determined colorimetrically by the standard Bratton-Marshall diazotization procedure. Correction was made for non-specific aryl amines, and a standard curve was included with each set of determinations. The method measures both the active antibiotic and inactive degradation products; the latter, however, are believed to be present only in a very low concentration, and in any event should remain relatively constant in amount during continuous fermentation.

The $\mathrm{pH}$ and percentage of total solids in the crude product were estimated at the time of sampling. The solids content was determined on a volume basis after centrifuging a $10 \mathrm{ml}$ sample for $10 \mathrm{~min}$ at $2000 \times g$.

Inoculum preparation. A culture of Str. venezuelae strain 05072 was obtained from Parke, Davis and Company; this strain can produce about $400 \mu \mathrm{g}$ of chloramphenicol per ml. Soil stocks of the organism were prepared from mature spores after growth 
on Anderson's sporulation agar. ${ }^{7}$ When a fermentation was begun, the spore stock was inoculated into $100 \mathrm{ml}$ of chloramphenicol medium II, ${ }^{7}$ modified as shown in Table $I$, in a $250 \mathrm{ml}$ Erlenmeyer flask. This was incubated on a rotary shaker at $28^{\circ} \mathrm{C}$ for $48 \mathrm{~h}$ and then used as inoculum for a larger $600 \mathrm{ml}$ culture in a 21 . flask. Three such flask cultures $(7 \cdot 5$ volumeper cent) were used to inoculate the fermentor.

Batch chloramphenicol fermentation. A series of batch fermentations were conducted to establish a reference picture in our equipment and with our operating procedures. The fermentor contained 20 l. of the modified chloramphenicol medium (Table I)

Table I. Composition of fermentation mediums

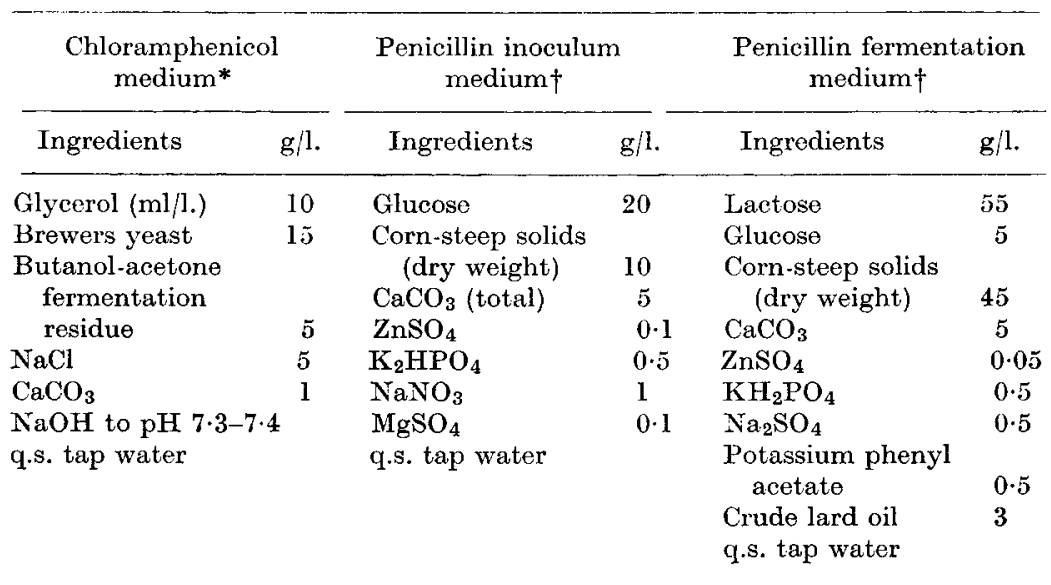

* Provided by Parke, Davis and Company.

$\uparrow$ Provided by Eli Lilly and Company.

with $1 \mathrm{ml}$ of General Electric No. 60 silicone-emulsion defoamer; air was supplied at a rate of $\frac{3}{4}$ volumes per volume of medium per min; the agitator was driven at a speed of $250 \mathrm{rev} / \mathrm{min}$, and the temperature was regulated at $28^{\circ} \mathrm{C}$.

Results of a representative batch chloramphenicol fermentation under these conditions are shown in Fig. 4. The maximum yield of $400 \mu \mathrm{g} / \mathrm{ml}$ in 4 days is relatively good for this strain. This particular experiment also was selected to demonstrate that even the early appearance of contamination sometimes has no apparent effect on chloramphenicol formation, a useful 


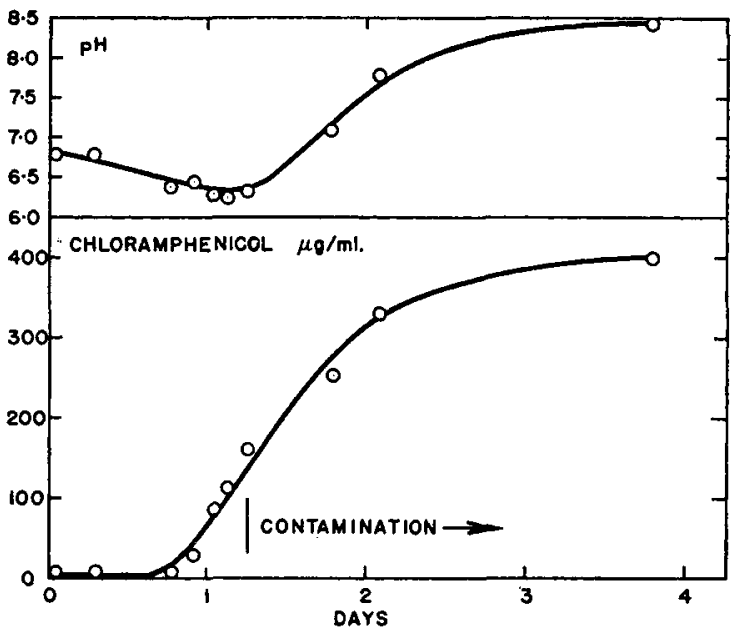

Fig. 4. Batch chloramphenicol fermentation

characteristic for continuous fermentation. However, the abnormal $\mathrm{pH}$ changes may have been caused by the contamination.

Continuous chloramphenicol fermentation. A continuous fermentation was begun in the same manner as a batch fermentation, allowed to progress for $24 \mathrm{~h}$, and then put on continuous feed with the same medium. Results of such a trial are shown in Fig. 5.

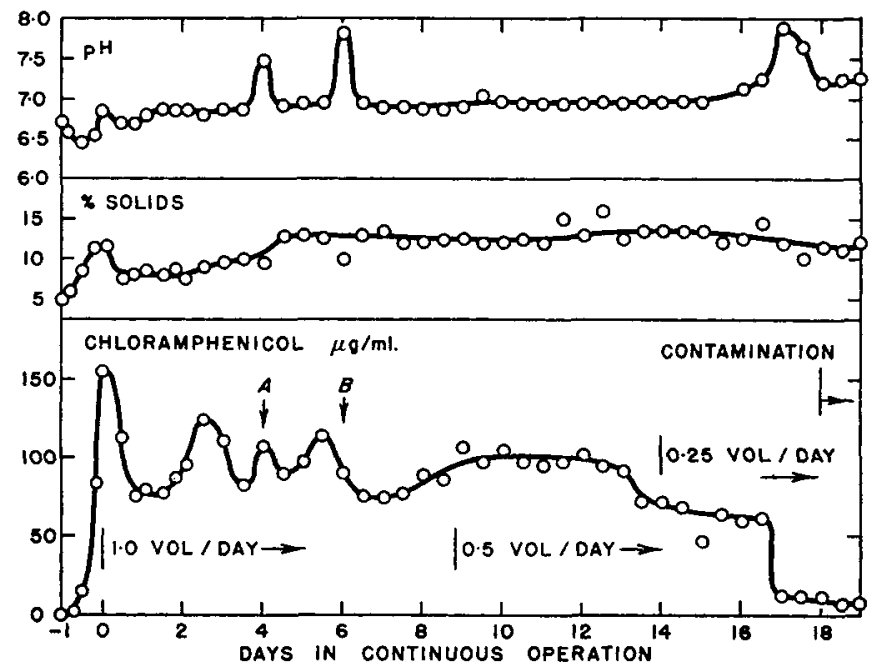

Fig. 5. Continuous chloramphenicol fermentation 
It was found that a dilution rate of 1.0 volume changes per day brought an initial drop from the higher batch level and then allowed maintenance of about one-quarter of the maximum batch yield. In three confirmatory experiments of shorter duration with the same dilution rate, yield levels again dropped initially and then were maintained at between 80 and $110 \mu \mathrm{g} / \mathrm{ml}$. As shown in Fig. 5, lower feed rates tended, if anything, to reduce the yield, although the $\mathrm{pH}$ and solids were relatively unaffected. The yield fluctuations observed in this and other continuous fermentations usually could be traced to some operational deviation and did not appear to be inherent in the method. For example, at points $A$ and $B$ on Fig. 5, the feed pump was inadvertently stopped for 5 and $10 \mathrm{~h}$, respectively. The appearance of contamination after $18 \frac{1}{2}$ days of continuous operation apparently had caused the yield to drop, and the experiment was terminated after 20 days.

The question was raised whether the drop in chloramphenicol production might be due rather to 'strain degeneration', especially since a lower yield level unpredictably accompanied the lowering of the feed rate in the third week. Accordingly, the organism was isolated from samples taken at the start of continuous operation as well as after 17 days in this experiment, and after 8 days in another experiment. Each of these cultures was then used to inoculate duplicate shaker flasks of medium and the resulting batch fermentations were analysed daily over a 10-day period. These results are given in Table II. Although the fermentations

Table II. Batch fermentation tests* of inoculums isolated initially and terminally from continuous chloramphenicol fermentations

\begin{tabular}{|c|c|c|c|c|c|c|c|c|c|c|c|c|}
\hline \multirow[b]{3}{*}{$\begin{array}{l}\text { Hours of } \\
\text { incubation }\end{array}$} & \multicolumn{6}{|c|}{ Tnoculum from Exp. $A$} & \multicolumn{6}{|c|}{ Inoculum from Exp. B (Fig. 5) } \\
\hline & \multicolumn{3}{|c|}{0 days } & \multicolumn{3}{|c|}{8 days } & \multicolumn{3}{|c|}{0 days } & \multicolumn{3}{|c|}{17 days } \\
\hline & pH & $\begin{array}{c}\% \\
\text { Solids }\end{array}$ & $\begin{array}{c}\mu g \\
\text { Chlor. }\end{array}$ & $\mathrm{pH}$ & $\begin{array}{c}\% \\
\text { Solids }\end{array}$ & $\begin{array}{c}\mu \mathrm{g} \\
\text { Chlor. }\end{array}$ & $\mathrm{pH}$ & $\begin{array}{c}\% \\
\text { Solids }\end{array}$ & $\begin{array}{c}\mu g \\
\text { Chlor. }\end{array}$ & $p H$ & $\begin{array}{c}\% \\
\text { Solids }\end{array}$ & $\begin{array}{l}\mu g \\
\text { Chlor. }\end{array}$ \\
\hline 48 & $7 \cdot 05$ & 5 & 3 & $6 \cdot 95$ & 5 & 2 & $7 \cdot 00$ & 4 & 0 & $7 \cdot 00$ & 5 & 3 \\
\hline 72 & $7 \cdot 20$ & 7 & 18 & $7 \cdot 18$ & 7 & 25 & $7 \cdot 1 \tilde{j}$ & 5 & 0 & $7 \cdot 20$ & 5 & 4 \\
\hline 96 & $7 \cdot 55$ & 8 & 36 & $7 \cdot 40$ & 9 & 37 & $7 \cdot 40$ & 7 & 16 & $7 \cdot 35$ & 7 & 17 \\
\hline 120 & $7 \cdot 75$ & 10 & 47 & $7 \cdot 65$ & 9 & 45 & $7 \cdot 65$ & 7 & 24 & $7 \cdot 50$ & 8 & 35 \\
\hline 144 & $7 \cdot 85$ & 11 & 50 & 7.90 & 10 & 5.5 & $7 \cdot 90$ & 8 & 40 & 7.85 & 8 & 44 \\
\hline 168 & $8 \cdot 10$ & 10 & 58 & $\div .95$ & 12 & 63 & 8.00 & 8 & 44 & $7 \cdot 95$ & 8 & 50 \\
\hline 192 & $8 \cdot 15$ & 11 & 68 & $8 \cdot 00$ & 15 & 72 & $8 \cdot 15$ & 8 & 48 & 8.05 & 9 & 51 \\
\hline 216 & $8 \cdot 30$ & 13 & 68 & $8 \cdot 20$ & 15 & 76 & 8.25 & 8 & 47 & $8 \cdot 30$ & 10 & 50 \\
\hline 240 & 8.45 & 14 & 67 & 8.40 & 15 & 72 & $8 \cdot 40$ & 8 & 37 & 8.40 & 10 & 45 \\
\hline
\end{tabular}

* Values given are averages of duplicate determinations.

$13+$ 
were in all cases more protracted and gave lower yields than usual, differences attributable to 'strain degeneration' from continuous culture were not apparent.

\section{Penicillin Fermentation}

In contrast to chloramphenicol, penicillin characteristically is formed after mycelial growth has approached its maximum, suggesting that the usual batch cycle is composed of two phases. ${ }^{32}$ Consequently, penicillin production under steady-state conditions should occur to best advantage in at least a two-stage system in which the growth and production phases could be separated and possibly enhanced individually. At best, singlestage continuous penicillin fermentation should represent only a low resultant of the two phases balanced at the steady-state. Although this outlook has generally prevailed, a recent note by Somerson and Nunheimer ${ }^{30}$ indicated that factors in the medium, rather than the mould mycelium, are responsible for the limitation of penicillin biosynthesis early and late in the batch cycle; thus, continuous penicillin fermentation in a single-stage system might be expected to be successful.

In further contrast to the chloramphenicol batch process, the penicillin fermentation is characterized by greater $\mathrm{pH}$ changes, a thick mycelial suspension, antibiotic activity primarily against Gram-positive bacteria, and a product that is unstable to both temperature and acid as well as to the penicillinase often produced by contaminating bacteria. The Penicillium mycelium tends neither to lyse nor to form spores in deep liquid culture.

A considerable amount of literature exists on the penicillin batch process, ${ }^{32}$ but continuous fermentations have been reported only in brief notes or as patents. Abraham ${ }^{1}$ and subsequently others $14,19,20,31$ have devised systems for continuously passing fresh medium beneath surface cultures or ${ }^{17}$ inside a Cellophane or porcelain tube on which the organism was grown. Attempts at single-stage continuous submerged fermentation have been made in shaving-packed generators 2,28 and in more conventional $30 \mathrm{l}$. fermentors, ${ }^{16}, 27$ in which yields of 500 to 800 units per $m l$ were maintained for 5 days. Several semi-continuous operations have been described in which a portion of a completed 
batch is retained in the fermentor as starter for a new cycle. ${ }^{10,26,33}$ A patent has been awarded for a two-stage continuous penicillin process in which overflow from a primary propagator is fed to a secondary fermentor. ${ }^{18}$

Assay procedures. The procedures for sampling and for determining $\mathrm{pH}$ and percentage of solids in the penicillin fermentations were the same as in the chloramphenicol process. Total carbohydrates were determined by the standard anthrone reaction. Penicillin was assayed by a paper-disc, zone-inhibition test similar to that recommended by the U.S. Food and Drug Administration. ${ }^{9}$ Commercial dehydrated media (Difco) with strain $\mathrm{H}$ of Staph. aureus (obtained from Eli lilly and Company) as the test organism were used in the assay procedure.

Inoculum preparation. A lyophilized spore suspension of Penicillium chrysogenum strain $\mathrm{N}-329$ was obtained from Eli Lilly and Company; this strain can produce about 2,000 units per $\mathrm{ml}$ but has a comparatively protracted fermentation period. Slant spore stocks of the mould were prepared by inoculating the lyophilized spores on Moyer's sporulation medium, ${ }^{21}$ incubating the cultures 8 days at $27^{\circ} \mathrm{C}$, and storing them in the refrigerator until needed. To begin a fermentation, spore suspension from one such tube was inoculated into 21 . Erlenmeyer flasks containing $500 \mathrm{ml}$ of Czapek-Dox corn-steep liquor medium ${ }^{25}$ modified for inoculum growth as shown in Table I. After incubation at 25 to $28^{\circ} \mathrm{C}$ on a shaker for 40 to $48 \mathrm{~h}$, three such cultures $(7 \cdot 5$ volume-per cent) were used to inoculate the fermentor.

Batch penicillin fermentation. As with the chloramphenicol experiments, a series of batch penicillin fermentations were conducted initially. The fermentation medium used (Table I) was typical of the production media reported in the literature, and included dried corn-steep liquor solids, potassium phenyl acetate, and lard oil. Air was supplied at a rate of 1.0 volume per volume of medium per min, the agitator was operated at a speed of $550 \mathrm{rev} / \mathrm{min}$, and the temperature was regulated at $26^{\circ} \mathrm{C}$.

Results of a representative batch penicillin fermentation under these conditions are shown in Fig. 6. The maximum yield of 1,800 units per $\mathrm{ml}$ in 7 days is representative for this strain, but the usual appearance of mycelial growth before the penicillin formation was not observed and the $\mathrm{pH}$ curve is atypical. 


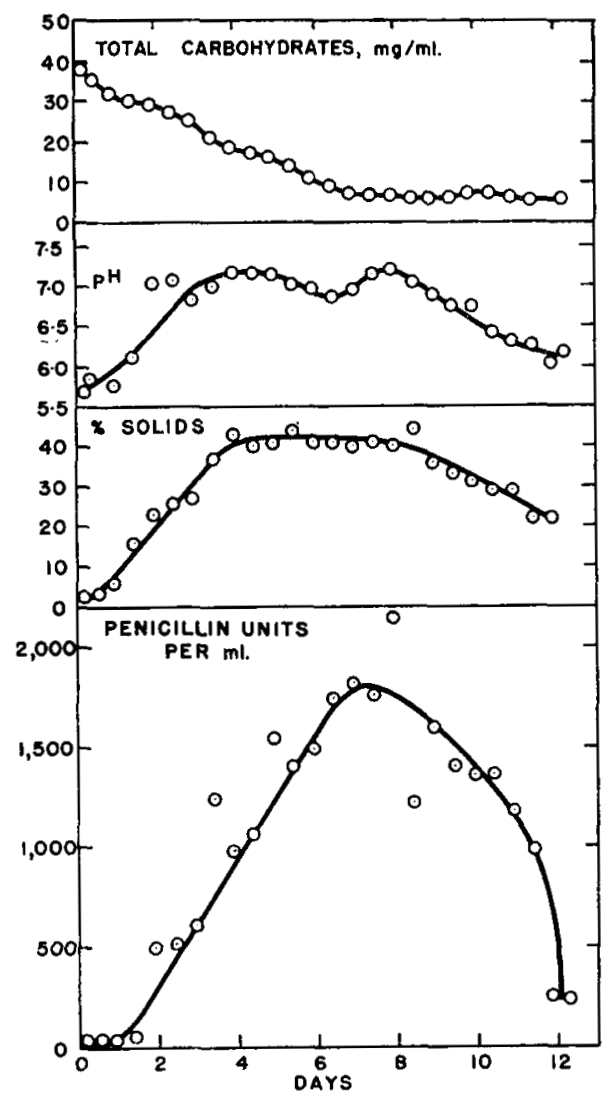

Fig. 6. Batch penicillin fermentation

Continuous penicillin fermentation. A continuous fermentation was begun in the same manner as a batch fermentation, allowed to progress for $48 \mathrm{~h}$, and then put on continuous feed with the same medium but with the lard oil fed separately. The first continuous run was made with a dilution rate of 1.0 volume changes per day, and as shown by the results in Fig. 7, both the mycelium and the penicillin level initially rose but subsequently were washed out after $4 \frac{1}{2}$ days of continuous operation. At point. $A$ on Fig. 7, the feed was stopped for $6 \mathrm{~h}$ and then was resumed at point $B$; at point $C$ the feed again was stopped for the duration 
of the run. 'The rises in antibiotic level that occurred at the points when the fermentation was allowed to proceed batch-wise indicated that the mould had not lost its ability to synthesize penicillin during the $4 \frac{1}{2}$ days of continuous culture. Two additional problems presented themselves in this first experiment: a heavy growth of eventually sporulating mycelium

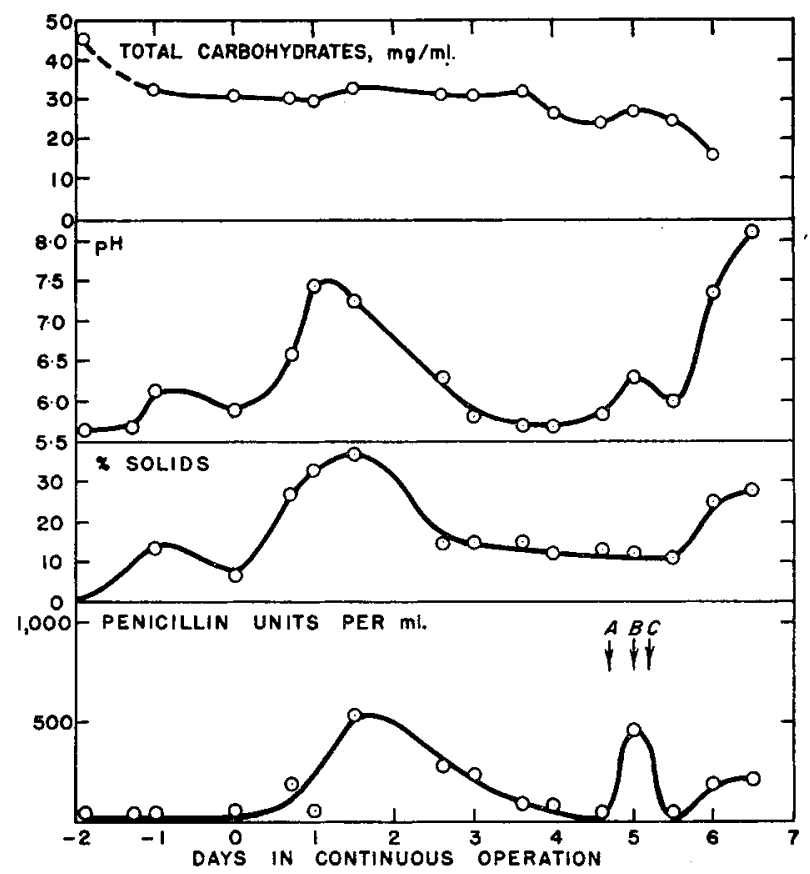

Fig. 7. Continuous penicillin fermentation at $1 \cdot 0$ volume changes per day

accumulated on the walls and baffles inside the fermentor (Fig. 8 ), and the mycelium in the effluent line after $4 \frac{1}{2}$ days plugged the back-pressure regulating valve. The first problem of mycelium accumulating inside the fermentor was never satisfactorily resolved; it appears to be an inherent limitation of continuous operation. The second problem was circumvented, or at least postponed, by substituting a vertically mounted Grinnell 'straightway' diaphragm valve for the conventional Saunders type in the pressure regulating unit. 
With this change in equipment and reduction of the medium feed rate to 0.5 volume changes per day, a second continuous penicillin fermentation (Fig. 9) was maintained successfully for 13 days, with a yield level between 400 and 1,000 units per $\mathrm{ml}$. Contamination occurred at 13 days, at which time the penicillin yield dropped and the run was terminated. Although the $\mathrm{pH}$ remained relatively steady during continuous operation, there

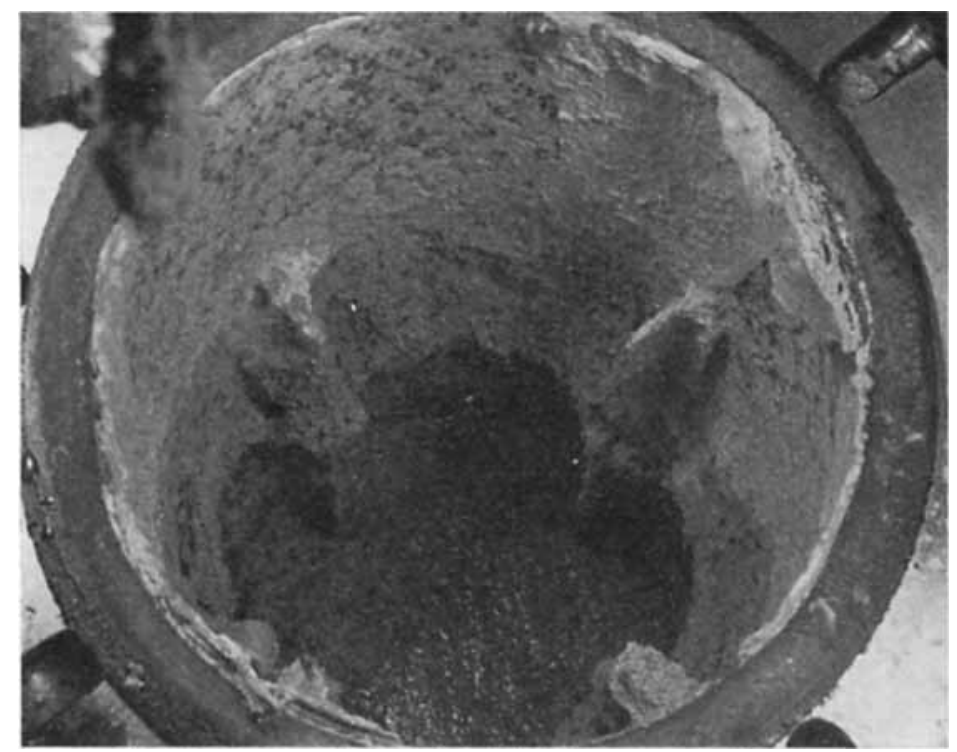

Fig. 8. View of accumulated mycelium in fermentor after 9 days of continuous penicillin fermentation

was considerable variation in the antibiotic level. As before, such fluctuations could usually be traced to an operational variable. For example, at points $A$ and $B$ on Fig. 9, the fermentor contents were observed to be foaming excessively; extra addition of antifoam agent was accompanied by an increased antibiotic level.

As shown in Fig. 10, a confirmatory run under the same conditions again reached and maintained for 3 days a penicillin level of 1,000 units per $\mathrm{ml}$. However, another operational variable again apparently caused reduced yields twice during the 8-day 
period of these operating conditions; at points $A$ and $B$ on Fig. 10, a valve was observed to be operating defectively and allowing steam condensate to enter the fermentor. In both this and the preceding run, the level of total carbohydrates steadily and unexplainably diminished during continuous fermentation. After

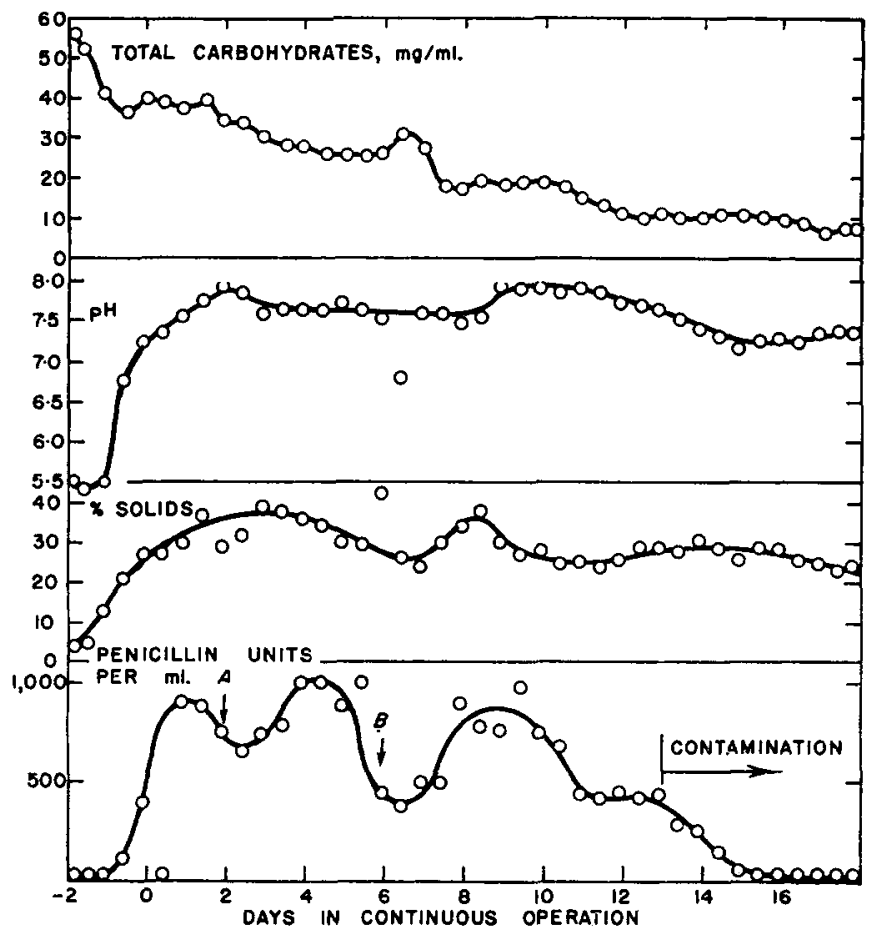

Fig. 9. Continuous penicillin fermentation at $0 \cdot \overline{5}$ volume changes per day

8 days, including recovery of the fermentation from the effects of the steam condensate, the medium being fed was changed so that 1.44 per cent glucose replaced the lactose originally employed. At the medium feed rate used, the glucose feed rate was 0.03 per cent per $h$. The use of glucose as a carbohydrate source at this feed rate was reported by Soltero and Johnson 29 to give better penicillin biosynthesis in batch fermentations. In our continuous operation, however, this change 
was associated with a gradual reduction in penicillin, solids, and carbohydrates and with a rise in $\mathrm{pH}$.

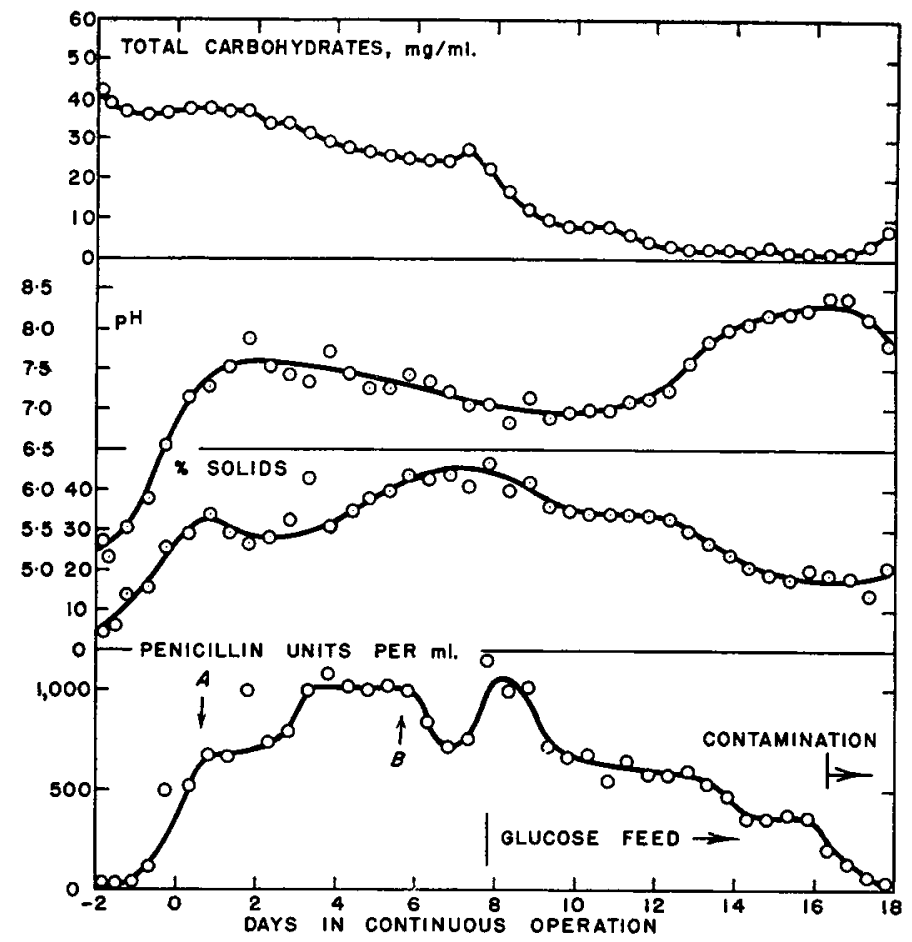

Fig. 10. Continuous penicillin fermentation at $0 \cdot 5$ volume changes per day, initially with lactose and then with glucose as the carbohydrate

\section{Discussion and Conclusions}

It appears that both chloramphenicol and penicillin can be produced by single-stage continuous fermentation processes. Using dilution rates of 1.0 and 0.5 volume changes per day, respectively, we were able to maintain both fermentations in the steady-state for periods in excess of 2 weeks with yields from $\frac{1}{4}$ to $\frac{1}{2}$ of the maxima we obtained in batch operation. In normal industrial practice, of course, production batches are harvested before the maximum is reached. Moreover, if the continuous yields are put on a daily production basis over a two-week operating period, they become roughly equal to or, in the case of 
penicillin, almost twice those achieved over the same period by batch operation with its characteristic shutdowns. It seems likely that further development of the continuous process could make its daily yield considerably more favourable. However, the relative practicability of the two types of operations depends on a number of features other than yields, so that further comparisons on the basis of the limited data so far available are not worth while.

A prominent feature of both of the continuous fermentations was the fluctuation, particularly in antibiotic level, with the passage of time. Finn and Wilson ${ }^{8}$ have reported that the continuous growth of yeast may induce a sinusoidal cycling of the population, which will normally decay exponentially. In our continuous antibiotic fermentations, the oscillations usually could be traced to some operational deviation and did not appear to be inherent in the operation. The occurrence of plateaux during periods of several days in some of the experiments suggested that better controls should permit the attainment of true steady-state fermentations with antibiotic production levelling at the peaks of the observed oscillations.

The finding that continuous fermentations of penicillin required a lower dilution rate than those of chloramphenicol was expected, since moulds usually grow more slowly than actinomycetes. Totally unexpected was the observation that a lower continuous dilution rate lowered rather than raised the chloramphenicol yield. Since this occurred at the end of a run and since replacement of the original population with a mutant strain has been predicted to be inevitable in continuous culture, ${ }^{22}$ the lowered yield level might have been due to 'strain degeneration' rather than to the lowered feed rate. An experiment to test this idea, however, indicated otherwise. Moreover, the result could not be traced to contamination or to the accumulation of toxic factors, since a return to a batch operation resulted in a rise in the yield. Since it was not accompanied by reduced mycelial solids, the reduced yield apparently was the result of a factor limiting only the biosynthesis of the antibiotic. The phenomenon requires further study.

The accumulation of Penicillium mycelium on surfaces inside the fermentor and within pipes and valves may prove to be an $13^{*}$ 
inherent and ultimate limitation of continuous production of penicillin. The situation in pipes and valves may be circumvented and postponed by appropriate modification of equipment. Conceivably, the mycelium retained within the fermentor may even contribute significantly to the steady-state yield level. Eventually, however, the resulting congestion will force cessation of the continuous operation, unless some method can be devised to prevent it.

Contamination with extraneous micro-organisms was common to all of the continuous fermentations conducted and represents another important limiting factor in successful continuous operation. Its control ultimately depends on refinements in equipment and handling, but certainly is possible. Although we operated aseptically for periods in excess of 2 weeks, other groups have conducted continuous bacterial cultures in a similar pilot plant for periods of several months. ${ }^{15}$ In almost every instance, we could trace contamination to the failure of some part of the equipment. Correction of such sources was and continues to be an important part of the development, e.g. replacing a fragile mechanical seal on the agitator shaft with a stuffing box. The larger, permanent equipment, accumulated experience, and possible integration of continuous sterilization with continuous fermentation should obviate many of the present problems of contamination if continuous fermentation becomes adopted in the fermentation industry.

Acknowledgement. The experimental work was supported by grants from Eli Lilly and Company and the Rackham School of Graduate Studies of the University. Much of the equipment was donated by Parke, Davis and Company.

Mrs. Jo-Ann Cherry, Mr. D. M. Gallup, and Miss Sara S. DeLong conducted the analyses.

\section{References}

1 Abraham, E. P., Chain, E., Fletcher, C. M., Gardner, A. D., Heatley, N. G., Jennings, M. A. and Florey, H. W. Lancet, 2 (1941), 177

2 Clifton, C. E. Science, 98 (1943), 69

3 Continuous Cultivation of Microorganisms, A Symposium_(1958), Prague; Czechoslovak Academy of Sciences

4 Continuous Culture Methods and Their Application. In Recent Progress in Microbiology, Symposia of VIIth Intern. Congr. Microbiol. (1958). Uppsala, Sweden; Almquist and Wiksells 
5 Distillers Co. Ltd., Ashton, H. W. and Holgate, J. (to Distillers Co. Ltd.) Brit. Pat. 660,145 (1951)

6 Distillers Co. Ltd., Jackson, C. J. and Milner, J. (to Distillers Co. Ltd.) Brit. Pat. 644,078 (1950)

7 Ehrlich, J., Anderson, L. E., Coffey, G. L. and Gottlieb, D. Antibiotics and Chemotherapy, 3 (1953), 1141

8 Finn, R. K. and Wilson, R. E. J. Agr. Food Chem, 2 (1954), 66

9 Food and Drug Administration, Compilation of Regulations for Tests and Methods of Assay of Antibiotic Drugs, Vol. I, Section 14la lh (1954). U.S. Department of Health, Education and Welfare, Washington, D.C.

10 Foster, J. W. and McDaniel, L. E. U.S Pat. 2,584,009 (1952)

11 Garibaldi, J. A. and Feeney, R. E. Industr. Engng. Chem. (Industr.), 41 (1949), 432

12 Gerhardt, P. and Bartlett, M. C. Advanc. Appl. Microbiol., 1 (1959), 215

13 Glazko, A. J., Wolf, L. M. and Dill, W. A. Arch. Biochem. (Biophys.), 23 (1949), 411

14 Haller, G. U.S. Pat. 2,519,902 (1950)

15 Herbert, D., Elsworth, R. and Telling, R. C. J. gen. Microbiol, 14 (1956), 601

16 Kolachov, P. J. and Schneider, W. C. U.S. Pat. 2,609,327 (1952)

17 Lewis, R. W. and Lucas, E. H. Science, 101 (1945), 364

18 Liebman, A. J. and de Becze, G. T. U.S. Pat. 2,524,200 (1950)

19 Lilly, C. H. U.S. Pat. 1,936,983 (1933)

20 Moor, W. A. Science, 102 (1945), 594

21 Moyer, A. J. and Coghill, R. D. J. Bact., 51 (1946), 57

22 Novick, A. Annu. Rev. Microbiol., 9 (1955), 97

23 Olive, T. R. Chem. Eng., 56 (1949), 107, 172

24 Oyaas, J. E., Ehrlich, J. and Smith, R. M. Industr. Engng. Chem. (Industr.), 42 (1950), 1775

25 Raper, K. B. and Thom, C. A Manual of the Penicillia (1949). Baltimore; Williams and Wilkins Co.

26 Schenley Industries, Inc. French Pat. 1,018,459 (1953)

27 Schneider, W. C., Andreasen, A. A. and Kolachov, P. J. 114th Meeting, Amer. Chem. Soc., p. 16A (1948). Portland, Ore.

28 Schwarze, W. Swiss Pat. 279,098 (1952)

29 Soltero, F. V. and Johnson, M. J. Appl. Microbiol., 2 (1954), 41

30 Somerson, N. L. and Nunheimer, T. D. Bacteriol. Proc. (Soc. Amer. Bacteriologists) (1958), p. 12

31 Stice, E. and Pratt, R. Science, 103 (1946), 535

32 Sylvester, J. C. and Coghill, R. D. Industrial Fermentations, Vol. 11 (1954). New York; Chemical Publishing Co., Inc.

33 Wakaki, S., Ishida, T., Yamaguchi, Y., Mizuhara, K., Masuda, K. and Kondo, Y. J. Antibiotics (Japan), 5 (1952), 622 